\title{
Linfoma anaplásico de grandes células associado a implante mamário: uma revisão narrativa
}

\author{
Large cell anaplastic lymphoma associated with breast implant: a narrative review
}

Linfoma anaplásico de células grandes asociado con implante de mama: una revisión narrativa

Ana Clara da Costa Silva ${ }^{1}$, Ana Paula Alves Pereira ${ }^{1}$, Bárbara Costa Bicalho ${ }^{1}$, Caroline Cançado Avelar $^{1 *}$, Eriane Damasia Faria de Almeida Abreu ${ }^{1}$, Giulia Costa Val Camarano ${ }^{1}$, Grace de Assis Costa $^{1}$, Isabela de Souza Freitas Martins ${ }^{1}$, Mariana Mortimer Magalhães ${ }^{1}$, José Tadeu Campos de Avelar'.

\section{RESUMO}

Objetivo: Realizar uma revisão narrativa atualizada acerca do Linfoma Anaplásico de Grandes Células Associado ao Implante Mamário (BIA-ALCL), por meio de discussões em torno das principais questões que envolvem a doença. Ademais, visa propagar informações que auxiliem médicos e estudantes da área a reconhecerem possíveis manifestações da neoplasia, bem como a atuarem no manejo de casos suspeitos. Revisão bibliográfica: $\mathrm{O}$ BIA-ALCL é um tipo de linfoma raro associado à texturização de implantes mamários. Entretanto, diante do elevado e crescente número de cirurgias estéticas e reconstrutivas realizadas no contexto brasileiro e mundial, a doença adquire relevância notável na prática médica. Sua forma de apresentação mais frequente ocorre tardiamente à colocação da prótese e pode ser diagnosticada por meio da ultrassonografia, com tratamento geralmente baseado na remoção cirúrgica do implante juntamente à cápsula fibrosa. Considerações finais: $O$ artigo buscou reunir evidências acerca da neoplasia para que aspectos relativos ao tema fossem elucidados, incluindo aqueles referentes a discussão médico-paciente sobre os riscos relacionados aos implantes texturizados, bem como à necessidade de acompanhamento periódico com o profissional.

Palavras-chave: Linfoma anaplásico de células grandes, Implantes de mama, Doenças mamárias.

\begin{abstract}
Objective: To conduct an updated narrative review about Anaplastic Large Cell Lymphoma Associated with Breast Implants (BIA-ALCL), through discussions around the main issues surrounding the disease. Furthermore, it aims to spread information that helps doctors and students in the field to recognize possible manifestations of the neoplasia, as well as to act in the management of suspected cases. Bibliographic review: BIA-ALCL is a rare type of lymphoma associated with texturing of breast implants. However, in view of the high and growing number of aesthetic and reconstructive surgeries performed in Brazil and worldwide, the disease acquires remarkable relevance in medical practice. Its most frequent form of presentation occurs long after the placement of the prosthesis and can be diagnosed by ultrasound, with treatment usually based on surgical removal of the implant together with the fibrous capsule. Final considerations: The article sought to gather evidence about the neoplasia so that aspects related to the theme could be elucidated, including those related to the doctor-patient discussion about the risks related to textured implants, as well as the need for periodic monitoring with the professional.
\end{abstract}

Keywords: Anaplastic large cell lymphoma, Breast implants, Breast diseases.

\footnotetext{
${ }^{1}$ Pontifícia Universidade Católica de Minas Gerais (PUCMG), Betim - MG.

*E-mail: avelarcaroline@yahoo.com.br

${ }^{2}$ Hospital Mater Dei, Belo Horizonte - MG.
} 


\section{RESUMEN}

Objetivo: Llevar a cabo una revisión narrativa actualizada sobre el linfoma anaplásico de células grandes asociado con implantes mamarios (BIA-ALCL), a través de debates sobre los principales problemas relacionados con la enfermedad. Además, su objetivo es difundir información que ayude a los médicos y estudiantes en el campo a reconocer posibles manifestaciones de la neoplasia, así como a actuar en el manejo de casos sospechosos. Revisión bibliográfica: BIA-ALCL es un tipo raro de linfoma asociado con la textura de los implantes mamarios. Sin embargo, en vista del alto y creciente número de cirugías estéticas y reconstructivas realizadas en el contexto brasileño y mundial, la enfermedad adquiere una relevancia notable en la práctica médica. Su forma de presentación más frecuente ocurre después de la colocación de la prótesis y puede diagnosticarse mediante ultrasonido, con un tratamiento generalmente basado en la extracción quirúrgica del implante junto con la cápsula fibrosa. Consideraciones finales: El artículo buscó reunir evidencia sobre la neoplasia para que se pudieran dilucidar aspectos relacionados con el tema, incluidos los relacionados con la discusión médico-paciente sobre los riesgos relacionados con los implantes texturizados, así como la necesidad de monitoreo periódico con el profesional.

Palabras clave: Linfoma anaplásico de células grandes, Implantes de mama, Enfermedades de la mama.

\section{INTRODUÇÃO}

O linfoma anaplásico de grandes células associado ao implante mamário (sigla em inglês: BIA-ALCL) é uma entidade nova que foi reconhecida pela Organização Mundial de Saúde (OMS) em 2016. O BIA-ALCL é um linfoma não Hodgkin raro que pode se desenvolver tardiamente após a implantação de próteses mamárias, acometendo a cavidade do seroma e/ou a cápsula fibrosa existente ao redor de implantes (JONES JL, et al., 2019 e SWERDLOW SH, et al., 2016).

O primeiro caso de BIA-ALCL foi relatado em 1997, e atualmente tem incidência estimada em 1 caso para 1-3 milhões de mulheres com implantes mamários por ano (JONES JL, et al., 2019). O BIA-ALCL tem como características morfológicas grandes células atípicas com citoplasma abundante eosinofílico, núcleos irregulares com nucléolos proeminentes. A imuno-histoquímica é positiva para CD30, $43-90 \%$ dos casos positivos para antígeno epitelial de membrana (EMA) e negativos para a enzima de linfoma anaplásico (ALK) (DE-AZAMBUJA AP, et al., 2020 e JONES JL, et al., 2019).

A fisiopatologia da doença ainda não está bem elucidada, sendo encontrado na literatura estudos que apontam tanto a possibilidade da formação do BIA-ALCL por meio de uma resposta inflamatória crônica relacionada a infiltração de células $T$ e da resposta imunológica da paciente, quanto estudos que atribuem o BIA-ALCL à resposta alérgica crônica mediada por interleucina 13 (IL13) produzida pelas células neoplásicas (MARRA A, et al., 2020).

Outros fatores importantes associados ao desenvolvimento dessa neoplasia são: texturização das próteses, composição do implante mamário, formação de um biofilme bacteriano em seu entorno, resposta imunológica e genética das pacientes (MARRA A, et al., 2020).

A apresentação clássica dessa neoplasia envolve seroma persistente, edema da mama, assimetria e dor (MARRA A, et al., 2020). Outras manifestações descritas em pacientes com BIA-ALCL são massa palpável, massa evidenciada em cirurgia, contratura capsular e rubor (KOLLING MLD, et al., 2019). Já que a principal manifestação da doença é o seroma, faz-se necessário que todas as pacientes que o desenvolveram após mamoplastia com prótese, sejam monitoradas e investigadas. Os exames iniciais devem ser: exame físico, mamografia ou ultrassom de mama, e biópsia por punção aspirativa por agulha fina (PAAF) do líquido seroso (MARRA A, et al., 2020).

Após confirmação diagnóstica, é necessário realizar o estadiamento utilizando a classificação de tumores malignos (TNM), onde se avalia a extensão tumoral, linfonodos acometidos e metástase. A partir disso, o manejo é definido e pode envolver quimioterapia, radioterapia e tratamento cirúrgico (CLEMENS MW e HORWITZ SM, 2019). 
Em todos os casos deve-se realizar a excisão cirúrgica da prótese e da cápsula fibrótica. Se pela biópsia a neoplasia for infiltrativa, a remoção cirúrgica é realizada de forma mais radical e o uso de terapias adjuvantes, como a quimioterapia citotóxica, pode se fazer necessária (MIRANDA RN, et al., 2014).

Quando diagnosticado de forma precoce, o BIA-ALCL é geralmente indolente, possui crescimento lento e tem excelente prognóstico (CLEMENS MW e HORWITZ SM, 2019). O BIA-ALCL é um desafio médico a ser compreendido, pois o uso de implantes mamários vem crescendo de forma exponencial em todo o mundo e consequentemente a chance de novos casos dessa neoplasia aumentam.

É importante abordar com as pacientes os riscos e benefícios do implante, elucidando inclusive a associação das próteses com o BIA-ALCL, além de pensar em estratégias de monitoramento desse grupo como a realização de mamografia sugerida pela Agência Internacional de Pesquisa em Câncer (MARRA A, et al., 2020). Diante disso, o artigo de revisão narrativa tem como objetivo estudar a associação entre o linfoma anaplásico de grandes células com os implantes mamários com base na literatura já existente.

\section{REVISÃO BIBLIOGRÁFICA}

\section{Epidemiologia}

Historicamente, a primeira cirurgia para inserção de implante mamário de silicone ocorreu em 1962, um ano após a criação do mesmo por dois cirurgiões plásticos americanos, Thomas Cronin e Frank Gerow, com colaboração da Dow Corning Corporation. Desde então, mais de 1,5 milhões de mulheres em plano mundial se submetem a mamoplastia de aumento anualmente, seja por motivos cosméticos ou para reconstrução mamária (GUNAWARDANA RT, et al., 2019).

Existem quatro formas distintas de Linfoma Anaplásico de Grandes Células (ALCL): Linfoma Anaplásico quinase positivo, Linfoma Anaplásico quinase negativo, ALCL cutâneo primário e ALCL associado ao implante mamário (FREEMAN AS e ASTER JC, 2020).

Primeiramente descrito em 1997, por J. A. Kreech Jr. e B. J. Creech, o BIA-ALCL foi incluído como uma nova entidade de neoplasias linfóides pela Organização Mundial de Saúde (OMS) em 2016. (JONES JL, et al., 2019; GUNAWARDANA RT, et al., 2019).

A Sociedade Americana de Cirurgiões Plásticos criou a PROFILE Registry (Patient Registry and Outcomes for Breast Implants and Anaplastic Large Cell Lymphoma Etiology and Epidemiology), para melhor entendimento da incidência, etiologia e epidemiologia do ALCL. Entre 2012 e 2018, 186 casos foram reportados no registro (NAHABEDIAN M e GUTOWSKI KA, 2020).

Segundo Jones et al. (2019), o risco cumulativo de desenvolver o Linfoma Anaplásico de Grandes Células mamário em uma população geral é de 0,35 a cada milhão na idade de 75 anos. Concomitantemente, o risco cumulativo em mulheres com implante seria de 29 a cada milhão na idade de 50 anos, aumentando para 82 a cada milhão aos 70 anos.

Segundo Marra et al. (2020), o risco é ainda maior em mulheres com implantes texturizados. Ainda segundo a autora, é importante ressaltar que não foi observado maior risco de BIA-ALCL em mulheres que foram submetidas à reconstrução mamária pós diagnóstico de câncer comparadas às cirurgias cosméticas.

Marra et al. (2020) cita algumas limitações na estimativa da incidência e risco de desenvolvimento do BIAALCL. Primeiramente, ela afirma que a prevalência de mulheres com implante mamário, e qual o tipo do implante, deve ser conhecido. Fatores, como o aumento do fenômeno do "turismo cosmético" e a falta de notificação em eventos adversos associados à prótese mamária, dificultam a obtenção de uma estimativa correta desse dado.

Uma segunda limitação seria a influência da maior conscientização no diagnóstico dessa entidade patológica no aumento do número de casos de BIA-ALCL. Por último, o subdiagnóstico e manejo clínico inapropriado podem levar a subestimação da real incidência da doença.

Segundo Clemens e Jacobsen (2020), o fato de que a condição é raramente vista nos centros médicos pode contribuir para o atraso no diagnóstico, que só vai levantar suspeitas durante a revisão do implante mamário devido a seroma persistente. 


\section{Apresentação clínica}

O linfoma anaplásico de grandes células pode se apresentar clinicamente de duas formas distintas, como um seroma ou uma massa tumoral. A primeira apresentação é determinada por um acúmulo de líquido entre o implante e a cápsula fibrosa, de ocorrência frequentemente tardia, com tempo médio de início entre 8 a 10 anos após a colocação da prótese (JONES JL, et al., 2019).

Esse tipo de efusão peri-protética pode estar relacionado à presença de edema local, assimetria e dor (MARRA A, et al., 2020). A segunda apresentação, menos comumente relatada, manifesta-se por meio de um tumor palpável, o qual apresenta células malignas que podem se infiltrar através da cápsula para o tecido adjacente, somando-se ao quadro, um possível acometimento linfonodal e sistêmico (JONES JL, et al., 2019).

Mulheres com apresentações de seromas tardios (após período superior a um ano de implantação da prótese mamária) não relacionados a outros diagnósticos diferenciais como aqueles decorrentes de traumas locais, infecções e ruptura do implante, devem ser avaliadas quanto à suspeita de ocorrência do BIA-ALCL (DePAOLA NEK e COGGINS H, 2019). A forma de apresentação como seroma, geralmente se caracteriza como uma neoplasia in situ e, por esse motivo, muitas vezes associa-se a um melhor prognóstico quando comparada à forma de apresentação tumoral infiltrativa (MARRA A, et al., 2020).

O BIA-ALCL desenvolve-se de forma unilateral na maioria dos casos, havendo poucos relatos documentados de acometimento bilateral das mamas (PÉREZ AT, et al., 2020). Outros tipos de manifestações clínicas incluem a presença de erupção cutânea e de contratura capsular, ainda que se apresentem com menor frequência.

Ademais, a literatura médica atual associa o desenvolvimento da neoplasia à implantes texturizados, o que sugere uma correlação, não apenas ao acaso, do linfoma anaplásico de grandes células com esse tipo de prótese, seja utilizada para fins estéticos ou reconstrutivos (DePAOLA NEK e COGGINS H, 2019).

\section{Patogênese}

A patogênese de BIA-ALCL, apesar de estudos, ainda não é completamente estabelecida. No entanto, hipóteses possuem como base de explicação a indução de resposta inflamatória crônica por próteses mamárias texturizadas, o que constitui potencial maligno das células T envolvidas. Rastogi, Deva e Prince (2018) indicam que a diferença da incidência da doença entre os implantes texturizados e os lisos está no fato de que os primeiros possuem maior área de superfície, além de apresentar relevos os quais permitem com maior facilidade a adesão de bactérias e a formação de biofilmes.

Em associação, a relação e resposta dos biofilmes aos implantes foram estudadas por Hu et al. (2015). Ao fazer testes em suínos e humanos, esses autores concluíram que ocorre um aumento da resposta dos linfócitos nos implantes texturizados em comparação aos lisos. Além disso, houve constatação de aumento de células $T$ e $B$ em relação à quantidade de bactérias $(H U H$, et al., 2015).

Na linha desses achados, Kadin et al. (2016), por meio de estudos com biomarcadores, evidenciaram que as células T de memória são guiadas por antígenos Th1/Th17 quando há estimulação bacteriana crônica no tecido capsular e no seroma circundante. Assim, este acontecimento, juntamente com a proliferação linfocitária, constituem os componentes iniciais e de progressão de BIA-ALCL.

Em 2018, outro estudo desses mesmos autores, mostrou que o microambiente da doença em questão tem em sua composição elevados níveis de interleucina 13 e $\mathrm{lgE}$, o que sugere que a resposta imune subjacente a BIA-ALCL possa ser relacionada a uma reação alérgica crônica (KADIN ME, et al., 2018).

Nesse contexto, há evidências de maior proporção de Rastonia spp (organismo Gram negativo) no microbioma capsular de pacientes com BIA-ALCL quando comparada a amostras de cápsulas não tumorais. Diante disso, Hu et al. (2016) chegaram à conclusão que a composição microbiana tem potencial de interferência na patogênese da doença, mas são necessários maiores esclarecimentos.

Em contrapartida, apenas essa explicação não é suficiente para definir uma etiologia para a doença. Estudos evidenciaram outros fatores, além do biofilme bacteriano, à exemplo da genética do paciente e sua resposta imune, como potenciais influenciadores (MARRA A, et al., 2020). 
Dessa forma, através de estudo in vitro, Lechner et al. (2012) comprovaram que, como em outras neoplasias, genes pró-apoptóticos e genes supressores de tumor das células de BIA-ALCL estão infra regulados em relação às células $T$ normais.

Ademais, esse mesmo estudo comprovou que células do linfoma em questão possuem receptores de interleucina 2 e interleucina 6 altamente expressos, bem como produção autócrina desta. Outro achado desses autores consiste na forte ativação do sinal de transcrição - Signal Transducer and Activator of Transcription 3 (STAT3), um dos principais precursores de BIA-ALCL sistêmica.

Estudos recentes publicados por Laurent et al. (2020) e Arianna et al. (2018) confirmaram a hipótese de envolvimento de fatores genéticos do hospedeiro. Esses autores encontraram mutações ativadoras de Janus Kinase and Signal Transducer and Activator of Transcription (JAK-STAT), o qual mostra-se envolvido com a proliferação, apoptose e diferenciação celulares. Foram identificadas, também, mutações recorrentes em TP53 e DNA metiltransferase (DNMT3A).

Referente ao exposto, pode-se concluir que os mecanismos elucidativos da patogênese de BIA-ALCL não estão completamente esclarecidos. Até o presente momento, as literaturas expõem diversas hipóteses e mecanismos envolvidos no processo, mas acordam na existência de inflamação crônica associada a implantes texturizados como principal base etiológica.

\section{Diagnóstico}

A investigação diagnóstica do BIA-ALCL inicia-se diante dos sintomas e sinais clínicos sugestivos dessa neoplasia (JONES JL, et al., 2019). As manifestações clínicas mais comuns incluem o aparecimento de seroma tardio ou inchaço da mama há pelo menos 1 ano, com média de 7 a 10 anos, após a colocação do implante mamário de superfície texturizada.

Diante dessas manifestações, realiza-se a avaliação ultrassonográfica das mamas, com o propósito de detecção da presença de derrames ou massas sugestivas para que, em seguida, haja a coleta do líquido e biópsia das massas mamárias e/ou dos linfonodos aumentados. A realização da ressonância magnética ou da tomografia computadorizada só é necessária caso os achados ultrassonográficos sejam indeterminados (CLEMENS MW e HORWITZ SM, 2019).

Após realização dos exames de imagem, deve ser feito a aspiração por agulha fina do fluido periprotético, com a retirada do máximo volume de líquido possível (no mínimo $50 \mathrm{ml}$ ). Se no ultrassom for detectado a presença de massa e/ou linfadenomegalia suspeita, torna-se necessário sua retirada por meio da biópsia.

Em seguida, as amostras devem ser enviadas para análise da morfologia celular. É feito o exame imunohistoquímico de CD30 e a citometria de fluxo, a fim de quantificar e caracterizar as células $\mathrm{T}$ presentes na amostra.

Vale ressaltar que a detecção de linfócitos CD30 positivos não é por si só patognomônico, uma vez que a expressão de CD30 é inespecífica. Portanto, para avaliação correta deve-se levar em consideração a correlação entre a história clínica e patológica da paciente (MARRA A, et al., 2020).

Na suspeita de acometimento sistêmico, como em pacientes que possuem invasão local agressiva ou metástase linfonodal, recomenda-se a realização de biópsia da medula óssea (CLEMENS MW e HORWITZ SM, 2019). Ademais, a realização da tomografia computadorizada por tomografia de emissão de pósitron (PET/CT) se mostra importante nos casos pré-operatórios de pacientes confirmados com BIA-ALCL, a fim de avaliar principalmente a presença de metástases e de massas capsulares associadas ou invadidas na parede torácica (MARRA A, et al., 2020).

Por fim, outros biomarcadores podem ser necessários para estabelecer o diagnóstico e excluir outras malignidades. Tais marcadores de expressão incluem o CD2, CD3, CD4, CD5, CD7, CD8, CD45 e o linfoma quinase anaplásico (ALK), sendo esse último sempre negativo nos casos de BIA-ALCL (CLEMENS MW e HORWITZ SM, 2019). A Figura 1 resume esquematicamente um algoritmo para gerenciamento de BIA-ALCL, o qual foi feito com base nas melhores evidências para alcançar o diagnóstico da doença. 
Figura 1 - Algoritmo proposto para diagnóstico de BIA-ALCL.

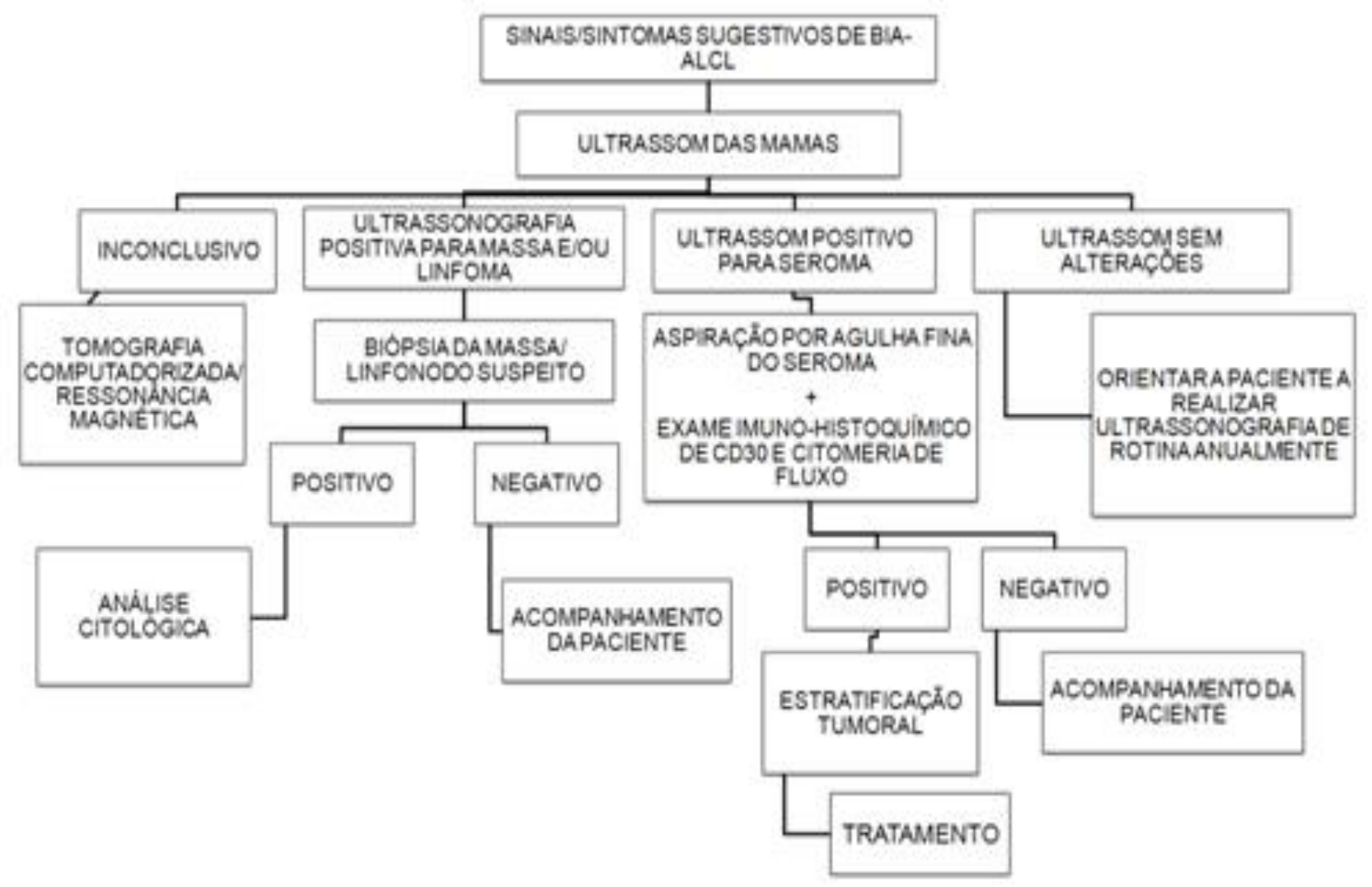

Fonte: Silva ACC, et al., 2020; dados extraídos de Marra A., et al., 2020 e Jones JL, et al., 2019.

Após análise citológica classifica-se o tumor de acordo com o estadiamento patológico com base no sistema TNM (Tabela 1), em que T consiste no tamanho do tumor, $\mathrm{N}$ indica a presença de nódulos linfonodais e $\mathrm{M}$ a presença de metástases.

Esse sistema mostra-se bastante útil para determinar a sobrevida global e a sobrevida livre de eventos, sendo, portanto, um sistema guia no estabelecimento do tratamento mais adequado para a paciente (JONES $\mathrm{JL}$, et al., 2019). De acordo com esse estadiamento, a maioria das pacientes (35\%) possui a doença em estágio T1N0M0, o que justifica, portanto, o bom prognóstico após tratamento adequado (DePAOLA NEK e COGGINS H, 2019).

Tabela 1 - Classificação TNM do Tumor Anaplásico de Células Grandes.

\section{T: Extensão do tumor}

T1: Presença de derrame ou seroma ao lado da cápsula

T2: Infiltração precoce na cápsula

T3: Agregados celulares infiltram-se na cápsula

T4: Infiltração além da cápsula

$\mathrm{N}$ : Presença de linfonodo

N0: Sem envolvimento linfonodal

N1: Envolvimento de um linfonodo regional

N2: Envolvimento de vários linfonodos regionais

\begin{tabular}{c}
\hline M: Metástase \\
\hline M0: Ausência de metástase \\
M1: Presença de metástase
\end{tabular}

Fonte: Silva ACC, et al., 2020; dados extraídos de DePaola NEK e Coggins H., 2019 e Clemens MW e Horwitz SM, 2019. 


\section{Tratamento}

Após o diagnóstico confirmado do BIA-ALCL, recomenda-se o acompanhamento com uma equipe médica multidisciplinar, em que oncologistas, patologistas, mastologistas e cirurgiões plásticos estarão inclusos. A decisão de tratamento é variável e dependente dos riscos individuais de cada paciente, assim, é aconselhável a realização de uma avaliação complementar, em que alguns testes laboratoriais poderão ser sugeridos, como o hemograma completo, painel metabólico completo, lactato desidrogenase (LDH) e a sorologia para hepatite B (CLEMENS MW e HORWITZ SM, 2019).

O diagnóstico oportuno, a remoção cirúrgica completa do implante com a cápsula fibrosa envolta e a excisão completa de possíveis massas encapsuladas residuais associadas são as abordagens essenciais para o tratamento do BIA-ALCL (CLEMENS MW e HORWITZ SM, 2019).

A excisão cirúrgica completa apresentou melhoria na sobrevida livre de outros eventos e prolongou a sobrevida global em comparação com as outras intervenções terapêuticas, como as cirurgias parciais, quimioterapia e a radioterapia.

Considerando que o BIA-ALCL não é uma doença relacionada ao parênquima mamário e que a cápsula pode drenar para múltiplos linfonodos, não há necessidade da realização de mastectomia radical ou biópsia de linfonodo sentinela (MARRA A, et al., 2020).

As doenças localizadas na cápsula IA-IIA, em sua maioria são tratadas apenas com a excisão cirúrgica completa, sendo este o atendimento padrão, possuindo baixo índice de recidiva. É importante que todas as tentativas de tratamento sejam feitas objetivando a ressecção cirúrgica total, a fim de evitar o uso de tratamentos adjuvantes (CLEMENS MW e HORWITZ SM, 2019).

Doença irressecável, doença residual pós excisão cirúrgica e doença localmente avançada, indicam a necessidade de tratamentos adjuvantes. Entretanto, os dados para o manejo desses pacientes ainda são limitados e não padronizados, devido à falta de estudos prospectivos comprovados que possam orientar o tratamento.

Dessa maneira, torna-se importante a discussão dos casos com a equipe médica multidisciplinar, considerando as comorbidades do paciente e a exposição anterior a algum outro tratamento (MARRA A, et al., 2020).

Às pacientes com doença residual local, margens positivas ou doença irressecável com invasão da parede torácica, recomenda-se o uso de radioterapia local com 24 a 36 Gray. A terapia sistêmica é utilizada em pacientes com doença em estágio II-IV (Lugano) ou IIB-IV (MDA).

Uma abordagem padrão seria a quimioterapia combinada com Antraciclina, ou uma combinação alternativa com Brentuximabe e Vedotina, sendo a adição de Brentuximabe considerada a terapia de primeira linha (CLEMENS MW, et al., 2019). O acompanhamento das pacientes deve ser realizado de 3 a 6 meses nos dois primeiros anos e posteriormente de acordo com a clínica (GUNAWARDANA RT, et al., 2019).

\section{Prognóstico}

O BIA-ALCL possui uma evolução majoritariamente indolente, de crescimento lento e localizado. $O$ prognóstico será decorrente do estágio em que a doença está, as comorbidades da paciente e outros fatores de risco. A excisão cirúrgica completa é a abordagem ideal para o tratamento da doença, de modo que doença retida ou irressecável indicam a necessidade de tratamentos adjuvantes (CLEMENS MW e HORWITZ SM, 2019).

O mau prognóstico está associado ao subconjunto clínico de pacientes que apresentam extensão de massa ou doença além da cápsula fibrosa externa. O diagnóstico em estágio I, que ocorre em $84 \%$ dos casos relatados, possui excelente desfecho, em que as pacientes conseguem a remissão da doença a partir da excisão cirúrgica completa e se mantêm sem recorrências (GUNAWARDANA RT, et al., 2019).

A taxa de recorrência da doença é 2,6 a 2,7 vezes maior em pacientes em estágios II e III em comparação àquelas em estágio I. Em pacientes com doença t4, após a excisão cirúrgica completa, a taxa de eventos é de 14,3\%, enquanto nas pacientes t1, t2 e t3 é 0\% (CLEMENS MW e HORWITZ SM, 2019). 


\section{Implicações psicológicas}

Podemos constatar, historicamente, que a instalação de um câncer afeta a mulher de forma profunda, seja por meio de sofrimento emocional e ansiedade, seja pela alteração da imagem corporal, autoconfiança e feminilidade. De acordo com DePaola NEK e Coggins H, (2019), nos casos de pacientes que desenvolvem o BIA-ALCL após a implantação de próteses mamárias, por questões reconstrutivas ou estéticas, o medo e a desordem psicológica são expressivas, já que a busca pela melhora da autoimagem é abalada com uma neoplasia rara em consequência do procedimento (MARRA A, et al., 2020).

É necessário que no pré-operatório o médico consiga expor informações consistentes sobre os riscos de complicações relacionadas ao implante de próteses mamárias e BIA-ALCL, a fim de evitar que pelas dúvidas e indecisões, as pacientes tenham percepções distorcidas do risco e acabem por usar a heurística de maneira a colaborar com as suas escolhas complexas e torná-las simples e, claro, por consequência, minimizar o sofrimento emocional. $\mathrm{O}$ objetivo do médico deve ser trazer, de forma consciente, protagonismo à paciente na tomada de decisão (MARRA A, et al., 2020).

Segundo Marra A, et al. (2020), as pacientes que tiveram o BIA-ALCL se sentem expostas ao risco de ocorrer outro tipo de câncer e, isso, transpõe um cenário de angústia. Além disso, os médicos têm um papel fundamental no apoio às pacientes com esse diagnóstico e na assistência de decisões conjuntas (DePAOLA NEK e COGGINS H, 2019). Nesse contexto, vale ressaltar que a reconstrução mamária deve ser avaliada, pois tem um grande valor emblemático, uma vez que se integra ao tratamento e significa não só o fim da luta contra o câncer, mas também o resgate da autoestima e do bem-estar emocional da mulher (MARRA A, et al., 2020).

\section{CONSIDERAÇÕES FINAIS}

A partir da análise dos estudos, pode-se observar que evidências apontam a associação entre implantes mamários e BIA-ALCL, mas não a relação de causalidade. A etiologia e os mecanismos fisiopatológicos não foram completamente esclarecidos, o que somados a baixa incidência, dificultam a elucidação do risco de desenvolvimento da doença e da elaboração de propedêutica para rastreio. Sugere-se estratégias de monitoramento com consultas anuais para revisão das próteses por meio do exame físico e/ou se surgirem anormalidades percebidas pelo paciente, com a complementação de exames de imagem. Diante do exposto, faz-se necessário a discussão entre médico e paciente dos riscos e benefícios do implante mamário, bem como de novos estudos que auxiliem no esclarecimento sobre o BIA-ALCL.

\section{REFERÊNCIAS}

1. ARIANNA DN, et al. Targeted next generation sequencing of breast implant-associated anaplastic large cell lymphoma reveals mutations in JAK/STAT signalling pathway genes, TP53 and DNMT3A. Br J Haematol., 2018; 180(5):741-744.

2. BATISTA BN, et al. Report of a case of anaplastic large cell lymphoma associated with a breast implant in a Brazilian patient. Rev. Bras. Cir. Plást., 2017; 32(3):445-449.

3. CLEMENS MW, HORWITZ SM. 2019 NCCN Consensus Guidelines on the Diagnosis and Treatment of Breast Implant-Associated Anaplastic Large Cell Lymphoma (BIA-ALCL). Aesthet Surg J., 2019; 39(Suppl_1):S3-S13.

4. CLEMENS MW, JACOBSEN E. Breast Implant - Associated Anaplastic Large Cell Lymphoma. UpToDate, 2020. Disponível em: <https:/www.uptodate.com/contents/breast-implant-associated-anaplastic-large-celllymphoma?source=history_widget>.

5. De-Azambuja AP, et al. Breast implant-associated anaplastic large cell lymphoma: a diagnostic challenge. Rev. Bras. Cir. Plást., 2020; 35(1):118-120.

6. DePAOLA NEK, COGGINS H. Breast Implant - Associated Anaplastic Large Cell Lymphoma: What We Know. J Adv Pract Oncol., 2019; 10(1):54-61.

7. FREEMAN AS, ASTER JC. Clinical manifestations, pathologic features, and diagnosis of systemic anaplastic large cell lymphoma. UpToDate, 2020. Disponível em: <https://www.uptodate.com/contents/clinical-manifestationspathologic-features-and-diagnosis-of-systemic-anaplastic-large-cell-lymphoma?source=history_widget>.

8. $\mathrm{HU} \mathrm{H}$, et al. Bacterial Biofilm Infection Detected in Breast Implant-Associated Anaplastic Large-Cell Lymphoma. Plast Reconstr Surg., 2016; 137(6):1659-1669.

9. $\mathrm{HU} \mathrm{H}$, et al. Chronic biofilm infection in breast implants is associated with an increased T-cell lymphocytic infiltrate: implications for breast implant-associated lymphoma. Plast Reconstr Surg., 2015; 135(2):319-329.

10. JONES JL, et al. National Co-ordinating Committee of Breast Pathology. Breast implant-associated anaplastic large cell lymphoma (BIA-ALCL): an overview of presentation and pathogenesis and guidelines for pathological diagnosis and management. Histopathology, 2019; 75(6):787-796. 
11. KADIN ME, et al. Biomarkers provide clues to early events in the pathogenesis of breast implant-associated anaplastic large cell lymphoma. Aesthetic surgery journal, 2016; 36(7):773-781.

12. KADIN ME, et al. IL-13 is produced by tumor cells in breast implant-associated anaplastic large cell lymphoma: implications for pathogenesis. Human pathology, 2018; 78:54-62.

13. KOLLING MLD, et al. Reação tecidual como causa do linfoma mamário. Rev. Bras. Cir. Plást., 2019; 34(0):92-95.

14. LAURENT C, et al. Gene alterations in epigenetic modifiers and JAK-STAT signaling are frequent in breast implantassociated ALCL. Blood, 2020; 135(5):360-370.

15. LECHNER MG, et al. Survival Signals and Targets for Therapy in Breast Implant-Associated ALK- Anaplastic Large Cell Lymphoma. Clinical Cancer Research, 2012; 18(17):4549-4559.

16. MARRA A, et al. Breast implant-associated anaplastic large cell lymphoma: A comprehensive review. Cancer Treat Rev., 2020; 84(101963):1-8.

17. MIRANDA RN, et al. Breast implant-associated anaplastic large-cell lymphoma: long-term follow-up of 60 patients. J Clin Oncol., 2014; 32(2):114-120.

18. NAHABEDIAN M, GUTOWSKI KA. Complications of Reconstructive and Aesthetic Breast Surgery. UpToDate, 2020. Disponível em: <https://www.uptodate.com/contents/complications-of-reconstructive-and-aesthetic-breastsurgery?source=history_widget $>$.

19. PÉREZ AT, et al. Linfoma anaplásico de células grandes e implantes mamarios: revisión sistemática de las casuísticas publicadas. Cir. Plást. Iberolatinoam., 2020; 46(1):25-36.

20. SWERDLOW SH, et al. The 2016 revision of the World Health Organization classification of lymphoid neoplasms. Blood, 2016; 127(20):2375-2390. 